\title{
Managing data entry of a large-scale interview project with optical scanning hardware and software
}

\author{
COLE BARTON \\ Davidson College, Davidson, North Carolina \\ and \\ CHRIS HATCHER, KAREN SCHURIG, PAUL MARCIANO, \\ KATHRYN WILCOX, and LOREN BROOKS \\ University of California, San Francisco, California
}

\begin{abstract}
A hardware and software system for the cost-effective preparation of interview data is described. Scantron optical scanning hardware and DataBlocks software were used to prepare ASCII data files from structured interviews and questionnaires. These data were then edited for analysis with SPSS-X Data Entry software. Three different processes for preparing interview data were compared. Time, personnel, hardware, and software costs were projected for each process, as well as comparative risks to the integrity of data. A scanning procedure saves time and money, and it reduces the risk of errors.
\end{abstract}

There are many reasons why clinical psychologists and other applied researchers use structured interviews and standardized psychometric instruments to collect research data. Although there is considerable variability in the methodological, psychometric, and clinical validity of these procedures, structured interviews and standardized paper-and-pencil tests are often the most appropriate research or clinical assessment procedures (Anastasi, 1988).

There are many possibilities for errors in the process of the collection and entry of data for statistical analysis with computers. Obviously, concerns about data entry and data preparation are related to the number of variables in a study. When data are entered manually into the computer, personnel time and salary costs are directly related to the size of the data set.

When data are entered, numbers can be misread and/or miscopied, repeated, or skipped. When coders must look back and forth between documents of differing formats, there are possibilities for recording the wrong data in the wrong locations. When there are many variables per subject and the data are not somehow identified on a terminal screen or on a posting sheet, to check for errors can be as time consuming as the entry process itself.

In addition to the integrity of data, there are practical concerns such as the expense of recruiting, training, and supporting data-entry personnel. The time to enter data into a computer data file may exceed the time it takes to collect it.

Correspondence should be addressed to Cole Barton, Department of Psychology, Davidson College, Davidson, NC 28036.
Contemplation of these concerns led us to consider hardware and software enhancements to our statistical software that would improve data entry. Our data-entry system consists of a Scantron scanner, DataBlocks Form Maker software, DataBlocks form-definition and scoring software, and SPSS-X Data Entry software (see Figure 1).

\section{Development of a Data-Entry System}

The variable template: SPSS-X Data Entry software. Data Entry is an add-on package for SPSS-X statistical software. It was designed primarily for the keyboard entry of data. The software enables a researcher to specify variables and value ranges, and to construct a screen template that functions as a data-entry form. The screen template makes it easier to enter the data correctly in the appropriate places (SPSS, 1988).

Although the data-entry screen can be configured to facilitate the keyboard entry of data, in our project it has primarily been used as a "template" for the ASCII code data files produced by the scanning process. A Data Entry "dictionary" was written, which defined each variable in our interview battery with its corresponding range and values. Once the ASCII data have been entered and checked with the Data Entry template, the data may be analyzed and manipulated with SPSS-X.

Optical scanner. Because keyboard entry of data is error-prone and time-consuming, we decided to have the data from our interviews transposed to forms that could be read by an optical scanner. An operator loads the scanner with forms and invokes software on the host computer that reads them. The major benefit of this equipment is the speed with which the scanner reads the forms and with which the data are saved to a file. This proce- 


\section{DATA REDUCTION AND CODING}

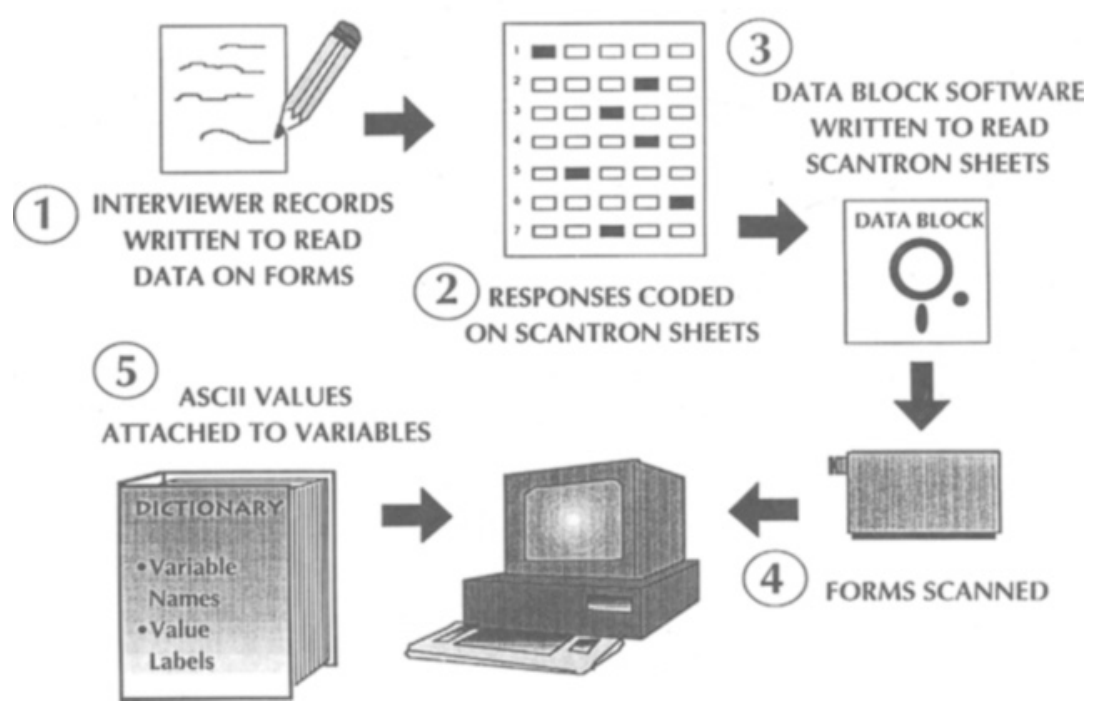

Figure 1. Scanner-supported data-entry process.

dure results in far fewer errors in comparison with manual data entry.

DataBlocks scanning software. Users can expand the capabilities of their scanning equipment if they explore the potential of software to make scanning more efficient and to do statistical analysis of a scanned data set. In addition to the form-reading function of the DataBlocks software, several pages of forms can be "linked" into one record, and different types of scores can be computed for different items.

The DataBlocks DB*Scan program tells the scanner the location of marks on the scanner page and how to interpret them, and it records the responses as ASCII code in a data file. Fields on the scanning sheet can be numeric or alphanumeric.

Parameters for test answers can be set, including: right or wrong; weights for the items; penalty or other scoring adjustments for guessing or marking critical items; creating subtest scores; or creating passing or criterion scores. All information about a test or set of tests can be saved in a file (DataBlocks, 1990).

There is DataBlocks software for the custom design and printing of forms that are compatible with the formdefinition software and Scantron hardware. The Form Maker software gives researchers almost unlimited flexibility in the construction of data-collection instruments that can be quickly and accurately scored.

\section{A Comparison of Three Data-Entry Procedures}

We compared three data-entry processes in terms of cost effectiveness. Process 1 was typical of keyboard entry of interview data like ours; Process 2 involved a scanner, form-definition software, and data-entry software.
Process 3 was the same as Process 2 with the addition of scannable questionnaire forms created by Form Maker software.

Some personnel problems contributed to our decision to investigate scanning procedures. We had difficulty finding qualified people willing to make long-term commitments to repetitious data-entry work. Those who do such work complain about the work a great deal, and they require intensive management to monitor errors, keep their motivation up, and create enough diversity to make data entry bearable.

The comparisons were based on subsamples of work that was actually performed using each of the three dataentry processes. We had people perform samples of each of the steps in each process, and we then projected how long it would take the work to be completed for our entire data set (see Table 1). These projections are probably conservative, since our professional staff agreed that the people who performed these tasks were superior in speed, grasp of instructions, and "correctness" in their work to most personnel trained for the specific task. For each process, the work was performed by the same two people. We made informal reliability comparisons of their pace and error rates to ensure comparability for purposes of projections.

Process 1. In Process 1, a data posting sheet on a large lined pad was created. Columns on the data posting sheets were derived from the items in our interviews. From the number of variables coded, the time taken to correct errors, and the time taken to prepare the forms, we projected that it would take $2,672 \mathrm{~h}$ to complete the posting sheets for our data set (over 10,000 variables for several hundred subjects). The length and complexity of the interview and 
Table 1

Time Costs in a Comparison of Data-Entry Processes

\begin{tabular}{|c|c|c|c|c|c|c|c|c|}
\hline \multicolumn{3}{|c|}{ Process 1} & \multicolumn{3}{|c|}{ Process 2} & \multicolumn{3}{|c|}{ Process 3} \\
\hline Step & Task & Hours & Step & Task & Hours & Step & Task & Hours \\
\hline 1 & $\begin{array}{l}\text { Create column-row } \\
\text { posting sheet }\end{array}$ & 2,672 & 1 & $\begin{array}{l}\text { Record interview data } \\
\text { on Scantron sheets }\end{array}$ & 1,720 & & $\begin{array}{l}\text { Interview data coded on } \\
\text { scannable interview forms }\end{array}$ & \\
\hline 2 & $\begin{array}{l}\text { Record interview data on } \\
\text { posting sheet }\end{array}$ & 2,438 & 2 & $\begin{array}{l}\text { Write Form Definition to } \\
\text { read scan marks }\end{array}$ & 150 & 1 & $\begin{array}{l}\text { Write Form Definition to } \\
\text { read scan marks }\end{array}$ & 150 \\
\hline 3 & $\begin{array}{l}\text { Keyboard entry of posted } \\
\text { data }\end{array}$ & 2,104 & 3 & Scan forms & 10 & 2 & Scan forms & 10 \\
\hline 4 & $\begin{array}{l}\text { SPSS-x system file } \\
\text { dictionary definition }\end{array}$ & 300 & 4 & $\begin{array}{l}\text { Data entry dictionary } \\
\text { definition }\end{array}$ & 240 & 3 & $\begin{array}{l}\text { Data entry dictionary } \\
\text { definition }\end{array}$ & 240 \\
\hline \multicolumn{2}{|c|}{ Total person hours: } & 7,514 & & & 2,120 & & & 400 \\
\hline
\end{tabular}

questionnaires were such that this step should actually save time over having the data entered directly from the datacollection forms.

On the basis of a subsample of work, we projected that recording the data from the interview sheets to the data posting sheets would take 2,438 h. Keyboarding the data in from the posting sheets to the computer would take another $2,104 \mathrm{~h}$.

Sampling the time taken to find and correct errors, time spent backtracking and place finding, and time spent sorting materials confirmed our suspicion that the steps involving the data posting sheets (even though they take up over two thirds of the hourly estimate) (1) result in faster data entry, as opposed to trying to enter data directly from a complex data set; and (2) are necessary for organization and monitoring of the data set as the data are being entered.

To use SPSS-X efficiently, the researcher not using Data Entry should define variable names and labels, as well as value names and labels, in a "system file." Variable-value names and labels need to be defined only once, but the process requires that the researcher locate the variables in appropriate columns, and write a format statement for each. This process is projected to take $300 \mathrm{~h}$ for a data set of this size.

The total number of hours to prepare the data for keyboard entry for a data set of our size is projected to be $7,514 \mathrm{~h}$. This process would not require the purchase of any additional equipment or software.

Process 2. Process 2 is the data-entry procedure that we are currently using, since at the time we began our project the DataBlocks Form Maker software was not available. As can be seen in Table 1, data entry begins by darkening the appropriate slots with No. 2 pencils on scannable forms. It would take $1,720 \mathrm{~h}$ to post the data equivalent to that described in Process 1.

A second step in the process is to write the "form definitions" with the DataBlocks software. The software allows the form-definition person to copy similar item formats and just key in appropriate matrix locations on the scanning sheet. In addition to the defining of the forms themselves, the form-definition phase consists of the "linking" of several forms into one case record. This step takes $150 \mathrm{~h}$. This process could go more quickly if there were no variability in the types of items and answers in a questionnaire.

For our data set, a conservative estimate of the time to scan sheets into data files would be $10 \mathrm{~h}$. Writing an SPSS-X Data Entry dictionary to attach variable names and labels, as well as value names and labels, to these scanned ASCII data would take $240 \mathrm{~h}$. The completion of Process 2 is therefore projected to take $2,120 \mathrm{~h}$.

Process 3. Even though DataBlocks' Form Maker was not available to us at the outset, the time that it would save is worth noting. Form Maker allows the researcher to design and print his or her own forms, and it saves the major data-entry labor. In addition, our preliminary experience with forms created with the Form Maker software shows that it greatly reduces errors in the posting of data, since interviewers and/or subjects can look at a form that prompts the type and location of responses. We project that if we had used the Form Maker product at the outset, we would have reduced the time necessary for our data-entry procedures to $400 \mathrm{~h}$.

Cost comparisons of the three data-entry processes. We projected $\$ 10$ per hour as an approximate salary for reasonably competent data-entry personnel. They offer a basis of comparability for the salary expenditures in these processes (see Table 2).

The cost for hardware is the price that we paid for the equipment we purchased.

There is considerable variability in the price of DataBlocks software. For nonprofit/educational organizations, the cost is approximately one tenth that for other corporations using mainframe computing equipment (see Table 2). We included the most expensive range of DataBlocks' form-definition software in our estimates, assuming that data sets as large as ours would need to be (as ours is) analyzed on mainframe equipment. We included the lower price range on the DataBlocks Form Maker 
Table 2

Financial Costs in a Comparison of Data-Entry Processes

\begin{tabular}{|c|c|c|c|c|c|}
\hline \multicolumn{2}{|c|}{ Process 1} & \multicolumn{2}{|c|}{ Process 2} & \multicolumn{2}{|c|}{ Process 3} \\
\hline Category & Cost & Category & Cost & Category & Cost \\
\hline Personnel $(\$ 10 / h)$ & $\$ 75,140$ & Personnel $(\$ 10 / \mathrm{h})$ & $\$ 21,200$ & Personnel $(\$ 10 / \mathrm{h})$ & $\$ 4,000$ \\
\hline Hardware & 0 & $\begin{array}{l}\text { Hardware: } \\
\text { Scanner }\end{array}$ & $\$ 4,000$ & $\begin{array}{l}\text { Hardware: } \\
\text { Scanner }\end{array}$ & $\$ 4,000$ \\
\hline Software & 0 & $\begin{array}{l}\text { Software: } \\
\text { Form Definition } \\
\text { Data Entry }\end{array}$ & $\begin{array}{r}\$ 8,000^{*} \\
\$ 1,000\end{array}$ & $\begin{array}{l}\text { Software: } \\
\text { Form Definition } \\
\text { Form Maker } \\
\text { Data Entry }\end{array}$ & $\begin{array}{r}\$ 8,000^{*} \\
\$ 800 \\
\$ 1,000\end{array}$ \\
\hline Totals: & $\$ 75,140$ & & $\$ 34,200$ & & $\$ 17,800$ \\
\hline
\end{tabular}

Note-Prices range from $\$ 600$ for IBM PC, nonprofit, to $\$ 8,000$ for mainframe, for-profit, $\quad$ "Large mainframes, forprofit corporations.

product, since we assume that forms can be printed with less expensive (PC) equipment. The SPSS-X Data Entry product is priced for use on a DEC MicroVAX II.

As can be seen in Tables 1 and 2, the differences in cost are substantial. In person-hours, entering a data set of this size by hand (Process 1) and getting it ready for data analysis can take over three times as long as when the data are scanned (Process 2). Process 3 takes only 5\% of the Process 1 time if the Form Maker product is used. Depending on personnel resources available, these factors may be even more salient in terms of producing timely data.

The dollar costs in these comparisons are substantial as well. Although most of the differences are caused by personnel costs $(\$ 75,140$ vs. $\$ 34,200)$, Process 2 leaves the researcher with equipment and software that could be used in future studies. We recognize that the differential may be less favorable to Process 2 if the cost of labor is low (e.g., graduate students or independent-study students), but settings in which one could use students would also be those in which the software costs would probably be lessened as well.

Finally, in discussions with colleagues who, like us, are struggling with data entry for large data sets, we are reminded of the practical and incalculable difficulties associated with making errors during data entry. In addition to personnel and time costs in research, the vulnerability to errors and other expense risks is substantial in most data posting, transposing, keyboarding, filing, and maintaining with hard copy. Some of the principal risks associated with each data-management strategy are summarized in Table 3.

It is worth noting again that Processes 2 and 3 offer several points for error-checking in the process of getting data from subjects to computer. One of the virtues of computers is that well-designed software is vigilant toward mistakes and prompts the researcher to make corrections. Staff members who are proficient with DataBlocks and Data Entry software assert that it is very difficult to proceed with data entry if data are omitted,

Table 3

Error Risks in a Comparison of Data-Entry Processes

\begin{tabular}{|c|c|c|}
\hline Process 1 & Process 2 & Process 3 \\
\hline $\begin{array}{l}\text { Likely errors inrow-column } \\
\text { row-sheets }\end{array}$ & $\begin{array}{l}\text { Misentry from interview form } \\
\text { to scan sheet }\end{array}$ & Forms must be handled carefully \\
\hline $\begin{array}{l}\text { Likely errors in transposing onto } \\
\text { row-column sheet }\end{array}$ & Forms must be handled carefully & \\
\hline \multicolumn{3}{|l|}{$\begin{array}{l}\text { Likely errors in misreading- } \\
\text { misentry from sheet to keyboard } \\
\text { to computer }\end{array}$} \\
\hline \multicolumn{3}{|l|}{$\begin{array}{l}\text { Difficulty keeping column } \\
\text { designation accurate in creating } \\
\text { system file dictionary }\end{array}$} \\
\hline $\begin{array}{l}\text { Difficulties in locating column } \\
\text { locations on terminal screen } \\
\text { (no labels) }\end{array}$ & & \\
\hline $\begin{array}{l}\text { Cost-ineffective highest paper } \\
\text { volume to store/file }\end{array}$ & & \\
\hline
\end{tabular}


or if data are in the wrong place or outside a specified range of values. In addition, each of these software packages helps the user.

\section{Summary}

In confronting the problems associated with preparing a large data set for computerized statistical analysis, we recognized the many time and personnel costs associated with getting data from the raw form created by our interviews into a form that would lend itself to multivariate statistical analyses. Besides the actual expense, we were concerned about the potential for (and even likelihood of) error at several critical junctures in the data-entry process.

It was cost-effective for us to use an optical scanner to read our data, largely because of the sophisticated software developed by DataBlocks. This software has allowed us the flexibility to accommodate many types of items in our methodology, to create appropriate variables of interest, to manipulate many different questionnaires and files, and even to perform some useful statistical analyses.

The Data Entry software facilitates editing the ASCII code produced in the scanning process. For our purposes, the congenial fit between Data Entry and our SPSS-X statistical program has further advantages.
Researchers with small budgets or few research assistants have been constrained from some forms of research because of the formidable costs of collecting and entering the data. As a result, many students' research training is compromised. More importantly, the number of important questions asked and answered is diminished.

In addition to the purchase of the equipment and software, there is some cost associated with staff development. The effective use of this system dictates that someone learn how to use the DataBlocks software in order to take full advantage of the system's capabilities.

Now that we have developed the system and the staff support for it, we have been able to pursue additional research and projects. The speed with which we can accomplish the collection and analysis of interview or questionnaire data has been as attractive to us and to funding agencies as the modest cost.

\section{REFERENCES}

Anastasi, A. (1988). Psychological testing (5th ed.). New York: MacMillan.

DATABLOCKS. (1990). DataBlocks installation and operations manual. Burbank, CA: Author.

SPSS. (1988). SPSS-X Data Entry II. Chicago: Author. 